\title{
A LIVING LIQUID CRYSTAL DISSECTED
}

\section{Jordi Garcia-Ojalvo}

Department of Experimental and Health Sciences, Universitat Pompeu Fabra

Barcelona Biomedical Research Park, Dr. Aiguader 88, 08003 Barcelona, Spain

Biofilms of rod-shaped bacteria self-organize in space and time in a manner reminiscent of nematic liquid crystals ${ }^{1}$. Like their abiotic counterparts, the global organization of these living liquid crystals is expected to emerge from the local interactions between their constituents - namely individual bacteria. But this hypothesis has not yet been validated in a systematic way, due to the difficulty of mapping all bacteria in a biofilm with sufficient spatial and temporal resolution as they proliferate. Now, writing in Nature Physics, Raimo Hartmann and colleagues have accomplished this feat by imaging biofilms of up to tens of thousands of cells.

In their most common realization, liquid crystals consist of elongated, anisotropic organic molecules that align with each other at low enough temperatures. This leads to a nematic phase characterized by long-range orientational order in a system that is nevertheless able to flow like a liquid. The physical mechanisms leading to the spontaneous alignment of the molecular orientations can be described by a generalized form of the intermolecular van der Waals potential, which accounts for the relative orientations of the interacting molecules and is minimized when the molecules are aligned ${ }^{3}$. This description allows us to dissect the role that microscopic interactions between the constitutive molecules play on the emergence of long-range order in the system.

Many bacterial species, such as those of the genera Escherichia, Bacillus and Vibrio, have elongated shapes that lead to anisotropic mechanical interactions when the cells live (and grow) in dense communities ${ }^{4}$. Although these interactions have been associated with local orientational ordering in small two-dimensional bacterial colonies ${ }^{4}$, the case for three-dimensional biofilms has proven more difficult to understand. Cells in bacterial biofilms are intricately embedded (see figure, bottom panel) in an extracellular matrix that plays an active role in the microscopic interactions that structure the community in space and time ${ }^{5}$. As in the case of abiotic liquid crystals, the emergence of local crystalline order in these systems can be better understood by identifying and parameterizing an effective cell-cell

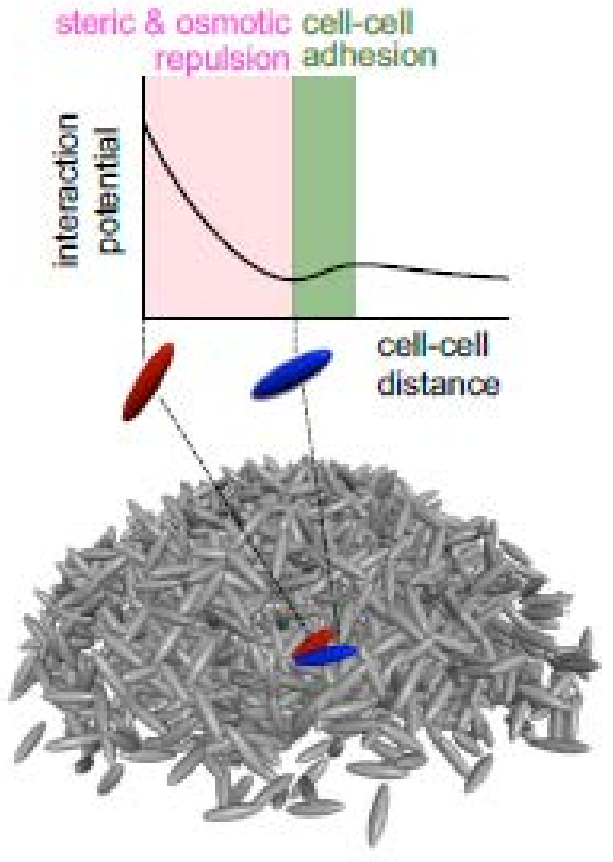

Figure: Identifying a cell-cell interaction potential for 3D biofilms. Schematic representation of a mid-sized biofilm of the type studied by Hartmann et al (bottom panel), and of the cell-cell interaction potential obtained in their paper (top panel), for a particular pair of cells. The specific parameter values that define the potential curve depend on the relative orientations of the two elongated bacteria. 
interaction potential that accounts for attractive and repulsive forces between neighbouring bacteria.

This is precisely what Hartmann et al accomplished ${ }^{2}$ in an experimental and computational tour de force that allowed them to monitor, in time and with single-cell resolution, the growth of biofilms for sizes up to 10,000 bacteria. In doing so, they succeeded in identifying and tracking all cells in the biofilm as they proliferated and moved through the self-secreted extracellular matrix, interacting with one another and shaping the global structure of the system. From this, they established an effective interaction potential and inferred its parameters by comparing experimental observations with a particle-based model of biofilm growth. This multifaceted approach allowed them to discern how relevant cell-cell mechanical interactions are to the local and global morphological properties of these cellular communities.

Using custom-made microfluidic flow chambers, Hartmann et al grew biofilms of the bacterium Vibrio cholerae and monitored their development in time, using high-resolution confocal fluorescence microscopy and a tailor-made 3D image segmentation method. For low flow rates, the biofilms have a hemispherical structure, which allowed the researchers to characterize the dynamics of different structural properties as a function of the distance to the centre of the biofilm in the basal plane.

Their results show a marked difference between the interior and periphery of the biofilm in several architectural properties, including the degree of alignment (nematic order), cell-cell distance, and radial and vertical orientations. Interestingly, the growth rate was seen to be basically homogeneous throughout the biofilm, in contrast with previous observations in Bacillus subtilis biofilms $^{6}$ - the difference being potentially due to the disparity between the biofilm sizes considered in the two studies. These structural observations were compared with a mutant strain lacking the protein $\mathrm{RbmA}$, the main mediator of cell-cell attraction in $\mathrm{V}$. cholerae biofilms. Strong differences with the wild-type strain — including lower nematic order, increased cell-cell distance and less-defined orientation - indicate the importance of cell adhesion in biofilm growth.

Next, Hartmann et al set out to identify an effective cell-cell interaction potential compatible with the structural properties they measured. Their proposed potential (see figure, top panel) depends on the distance between the cells and on their relative orientation. It contains a repulsion term arising from both steric and osmotic forces, a strong attraction term resulting from direct adhesion through RbmA, and a mean-field background term in the form of the Woods-Saxon potential. The latter is commonly used in nuclear physics to describe nucleon interaction, but here it represents weak cell-cell binding through Vibrio polysaccharide, one of the main components of the extracellular matrix.

To determine the parameters of the potential, individual-based simulations of growing biofilms were performed using an efficient parallel implementation of the model in graphics processing units. The authors checked that simulations with the best-fit parameters reproduced structural features that weren't included in the cost-minimization procedure used in the fitting. They were then able to predict the structural properties of biofilms comprising more than 300 cells - larger than those used to fit the potential. Taken together, these results support the hypothesis that the 
orientational order and global morphology of the biofilms studied can be explained solely in terms of mechanical interactions between the cells in the population.

Finally, Hartmann et al used the insights gained from their combined experimental and computational approach to examine how the morphology of their biofilms was altered by external flow fields, with shear rates varying over three orders of magnitude, mimicking the variety of environments encountered by these cellular communities in real-life situations. Their observations revealed that the mechanical cell-cell interactions they uncovered play a key role in determining the structural response of biofilms to applied external fields - not unlike what happens in nonliving liquid crystals.

As is usually the case in challenging problems like this one, the approach of Hartmann et al has some limitations. Perhaps most obvious is the assumption that the parameters of the effective interaction potential are uniform throughout the biofilm and through time, a premise that can be expected to break down in later stages of biofilm development, when the community reaches sizes on the order of millions of cells. In that sense, it would be interesting - although clearly a formidable task — to use this approach to understand the large-scale morphological features that arise in larger biofilms, such as wrinkles ${ }^{7}$ and channels $^{8}$, which are so far unique to living liquid crystals.

\section{References}

1. Wong, G.C. (2016). Three-dimensional architecture of Vibrio cholera biofilms. Proceedings of the National Academy of Sciences, 113(14), 3711-3713.

2. Hartmann, R. et al (2018). Emergence of three-dimensional order and structure in growing biofilms. Nature Physics, in press.

3. Gelbart, W.M. (1982). Molecular theory of nematic liquid crystals. The Journal of Physical Chemistry, 86(22), 4298-4307.

4. Volfson, D., Cookson, S., Hasty, J., \& Tsimring, L.S. (2008). Biomechanical ordering of dense cell populations. Proceedings of the National Academy of Sciences, 105(40), 15346-15351.

5. Drescher, K. et al (2016). Architectural transitions in Vibrio cholerae biofilms at single-cell resolution. Proceedings of the National Academy of Sciences, 113(14), E2066-E2072.

6. Liu, J. et al (2015). Metabolic co-dependence gives rise to collective oscillations within biofilms. Nature, 523(7562), 550.

7. Asally, M. et al (2012). Localized cell death focuses mechanical forces during 3D patterning in a biofilm. Proceedings of the National Academy of Sciences, 109(46), 1889118896.

8. Wilking, J.N. et al (2013). Liquid transport facilitated by channels in Bacillus subtilis biofilms. Proceedings of the National Academy of Sciences, 110(3), 848-852. 